\title{
Fine Motor Skills Relate to Visual Memory in Autism Spectrum Disorder
}

\author{
Yasuko Funabiki ${ }^{1}$, Tadao Mizutani ${ }^{1} \&$ Toshiya Murai $^{1}$ \\ ${ }^{1}$ Department of Psychiatry, Graduate School of Medicine, Kyoto University \\ Correspondence: Yasuko Funabiki, Department of Psychiatry, Graduate School of Medicine, Kyoto University, \\ 54 Kawahara-cho, Shogoin, Sakyo-ku, Kyoto, 606-8507, Japan. Tel: 81-757-513-373. E-mail: \\ funaysk@kuhp.kyoto-u.ac.jp
}

Received: October 26, 2014

Accepted: February 19, $2015 \quad$ Online Published: April 9, 2015

doi:10.5539/jedp.v5n1p88

URL: http://dx.doi.org/10.5539/jedp.v5n1p88

\begin{abstract}
Memory function is an important determinant of behavioral manifestations and social adaptations in individuals with autism spectrum disorder (ASD). While a number of studies have examined memory function in people with ASD, whether impaired memory is characteristic of ASD is unclear. This controversy is likely because of the heterogeneity of the ASD population. However, few studies have investigated the relationship between memory function and the severity of various autistic symptoms. Therefore, to assess such relationships, we used the Wechsler Memory Scale-Revised and the Multi-dimensional Scale for Pervasive developmental disorder and Attention deficit/hyperactivity disorder, which encompasses symptoms comorbid with ASD, to assess 36 individuals with high-functioning ASD. Participants showed average performance in the memory battery. Interestingly, scores reflecting fine motor skills were significantly associated with visual memory performance. That is, individuals with ASD who exhibit impaired fine motor skills showed poor visual memory. This finding implies that memory variability may be associated with developmental trajectory in people with ASD.
\end{abstract}

Keywords: autism spectrum disorder, fine motor skills, memory, multi-dimensional scale for pervasive developmental disorder and attention-deficit/hyperactivity disorder, Wechsler Memory Scale-Revised

\section{Introduction}

Autism spectrum disorder (ASD) is characterized by impairments affecting social interaction and communication, as well as restricted or repetitive behaviors and interests. Although ASD is defined according to these core behavioral features, individual variability in the characteristics of the symptoms, including comorbidity, is prominent among individuals with ASD (Matson \& Nebel-Schwalm, 2007; Funabiki, Kawagishi, Uwatoko, Yoshimura, \& Murai, 2011). This variability may be an important consideration when assessing medical and social support available to this population. Among the individual variability in ASD symptoms, impaired memory is often anecdotally reported. For example, Steel, Gorman, and Flexman (1984) reported underperformance in sentence and visual memory tasks and difficulty with free recall in ASD patients. Conversely, excellent rote memory has also been observed in this population (Kanner, 1943; Bemporad, 1979).

Many researchers have systematically investigated visual and verbal memory function in people with ASD. Researchers have generally agreed that both children and adults with ASD perform comparably to controls on verbal memory tasks, including word recognition tasks (Bennetto, Pennington, \& Rogers, 1996; Bowler, Gardiner, \& Grice, 2000), a word paired association task, a short story recall (Williams, Goldstein, \& Minshew, 2005b; Ambery, Russell, Perry, Morris, \& Murphy, 2006), and verbal working memory tasks (Siegel, Minshew, \& Goldstein, 1996; Koshino et al., 2005; Spek, Scholte, \& Van Berckelaer-Onnes, 2008). In terms of visual memory, some studies have reported that individuals with ASD have no difficulties with visual recognition or spatial working memory tasks (Bennetto et al., 1996; Renner, Klinger, \& Klinger, 2000; Ozonoff \& Strayer, 2001), whereas others reported difficulties in a task where participants retrieved and drew designs (Williams, Goldstein, \& Minshew, 2006), a visuospatial working memory task (Steele, Minshew, Luna, \& Sweeney, 2007), and the Spatial Span task from the Wechsler Memory Scale-III (Williams, Goldstein, Carpenter, \& Minshew, 2005a). Thus, visual memory appears to be either normal or poor in people with ASD. This contrasts with suggestions that children with ASD are visual learners rather than auditory learners (Cohen, 1998). 
This inconsistency might be due to the individual variability of symptom characteristics in ASD. However, few studies have investigated the relationship between memory performance and the severity of autistic symptoms. Steele et al. (2007) administered a spatial working memory task and examined the relationship between task performance and the severity of ASD symptoms, as measured by algorithm scores from the Autism Diagnostic Observation Schedule-Generic and the Autism Diagnostic Interview-Revised. They did not find any significant correlations. Conversely, Dawson, Meltzoff, Osterling, and Rinaldi (1998) conducted a delayed non-matching to sample task (DNMS) and found correlations between DNMS performance and the severity of autistic symptoms. Thus, the relationship between specific ASD symptoms and visual working memory is unclear.

Inter-individual variability of visual memory performance in people with ASD might not be caused by ASD itself, but by comorbid features. We previously developed a comprehensive assessment of behavioral symptoms termed the multi-dimensional scale for pervasive developmental disorder and attention-deficit/hyperactivity disorder (MSPA). The MSPA not only encompasses behavioral features in the social domain, but also accounts for other associated features, including motor skills (Funabiki et al., 2011). In this study, we used the MSPA to assess a wide range of characteristics of individuals with ASD, as well as a well-known memory battery, the Wechsler Memory Scale-Revised (WMS-R: Wechsler, 1987), for visual and verbal memory assessment. Thus, we investigated possible correlations between the behavioral features of ASD, including comorbidities, and memory performance, with respect to individual differences.

\section{Method}

\subsection{Participants}

We recruited individuals with ASD who had no physical complications or psychosis from outpatient clinics or colleges in our city. We chose participants with a full-scale intelligence quotient (IQ) of 70 or higher to omit confounds due to low IQ scores. All participants gave written informed consent. Trained psychiatrists confirmed diagnoses of pervasive developmental disorder (PDD) according to the criteria in the DSM-IV-TR (American Psychiatric Association, 2000), and we regarded PDD as ASD. IQ was assessed using the Wechsler Adult Intelligent Scale-Third Edition (Wechsler, 1997). We used data from the Wechsler Intelligence Scale for Children-Third Edition (Wechsler, 1991) for one 16-year-old participant. In total, 36 individuals with ASD (three with high-functioning autism, nine with Asperger syndrome, and 24 with pervasive developmental disorder not otherwise specified) participated in this study (Table 1). The mean age and standard deviation were 30 and 9 , respectively. Twenty-four participants were male and 12 were female. This study was approved by the Ethics committee at our University Hospital.

Table 1. Demographic data of participants

\begin{tabular}{lll}
\hline & Mean \pm SD & Range \\
\hline Number (male: female) & $36(24: 12)$ & \\
Age & $30.4 \pm 9.0$ & $16-50$ \\
Full IQ & $99.14 \pm 14.71$ & $74-134$ \\
VIQ & $104.56 \pm 14.75$ & $72-134$ \\
PIQ & $91.89 \pm 16.15$ & $56-129$ \\
\hline
\end{tabular}

\subsection{Materials}

The WMS-R (Wechsler, 1987) is a comprehensive memory battery that is normalized for adolescents and adults. It consists of five memory indexes (General, Verbal Memory, Visual Memory, Attention/Concentration, and Delayed Recall). The Verbal Memory index tests consist of Verbal Paired Associates and Logical Memory, and the Visual Memory index tests consist of Figure Memory, Visual Paired Associates, and Visual Reproduction. The Attention/Concentration index assesses working memory or the attentive aspect of memory, and includes Digit Span, Mental Control, and Visual Memory Span tests. The Delayed Recall index assesses the recall of Verbal Paired Associates, Logical Memory, Visual Paired Associates, and Visual Reproduction after 30 minutes. We converted the raw scores for each subtest into summed index scores. This enabled the construction of a 
profile from which relative levels of performance on the various memory functions could be directly compared, irrespective of participant age.

The MSPA (Funabiki et al., 2011) is a comprehensive assessment tool for PDD (ASD) and attention-deficit/hyperactivity disorder (ADHD). While ASD has a wide variety of symptoms and individual differences, the MSPA can be used to assess core ASD features, as well as other factors, such as inattention, motor problems, and sleep issues. It includes 14 domains, encompassing a range of clinical and behavioral features. Five of the domains (Communication, Social Adaptation, Empathy, Restricted Interests/Behavior, and Stereotyped/Repetitive Motion) represent core features of ASD. The Gross Motor domain assesses gross motor abilities, such as walking pattern, balance, and athletic performance. The Fine Motor domain assesses fine motor skills, such as handwriting, drawing, and dressing. Three of the domains (Inattention, Hyperactivity, and Impulsivity) address features of ADHD. Four of the domains (Sensory, Sleep Cycle, Learning, and Language Development) concern features that are often comorbid with ASD. "Learning" refers to Learning Disorder (LD). Each domain is assessed on a scale of one to five in half points, as follows: one (no sign), two (somewhat evident but no need of support), three (evident with a special need for supervision in a group setting), four (evident with a special need for supervision in a specialized group setting), five (difficulty even with full-time specialized group support, and special needs evident in individual life). Symptom severity was defined using the DSM-IV-TR for all domains other than Sleep Cycle. In short, a score of 3, 4, and 5 denotes mild, moderate, and severe symptoms, respectively. Thus, populations with typical development usually have scores below 3 . The rater assembled information on behaviors from birth to the present using medical records and interviews with the patients, parents, and teachers of the participants. The rater then evaluated the severity of symptoms and excluded the influence of physical disease, handicaps, and specific environments. Thus, we administered the MSPA to assess clinical and behavioral characteristics of participants, assuming an average social environment. This scale had sufficient reliability among trained staff (Funabiki et al., 2011).

\subsection{Procedure}

Five trained psychologists took charge of the WMS-R. One of the staffs conducted the test according to the administration manual (Wechsler, 1987; Sugishita, 2001). Afterwards, one of eight MSPA-trained staffs, who was not informed of the WMS-R results, administered the MSPA. Other trained staffs discussed and confirmed each MSPA score. Before administering the MSPA, each examiner had achieved a set reliability standard.

\subsection{Analysis}

We calculated the WMS-R index scores according to the administration manual (Wechsler, 1987; Sugishita, 2001). We conducted a correlation analysis between the index scores from the WMS-R and each domain in the MSPA. We used the two indexes of Verbal and Visual Memory for the correlation analyses, because the other three indexes are mixed scores containing various factors, such as concentration or delayed recall. We adopted a threshold of $\mathrm{p}<0.01$ (uncorrected) for correlations between symptoms and memory performance, and were careful in our interpretations, as the results were of an exploratory nature (please see the discussion section). We used the Pearson product-moment correlation coefficient and SPSS for our correlation analysis.

\section{Results}

As shown in Table 1, the mean of the full-scale IQ in this study was 99.14, which is very close to 100 . Additionally, all five of the main index scores in the WMS-R approached 100, as shown in Table 2. The obtained MSPA scores are shown in Table 3. Because the participants in this study were diagnosed with ASD, the scores of the core features were high. However, the other domains had wide ranges.

Table 2. Memory performance scores from the WMS-R

\begin{tabular}{lll}
\hline & Average \pm SD & Range \\
\hline General Memory & $97.69 \pm 19.68$ & $50-133$ \\
Verbal Memory & $98.75 \pm 18.94$ & $65-133$ \\
Visual Memory & $97.44 \pm 16.44$ & $50-120$ \\
Attention/Concentration & $96.67 \pm 15.14$ & $50-120$ \\
Delayed Recall & $97.22 \pm 17.20$ & $50-129$ \\
\hline
\end{tabular}


Table 3. Severity of ASD and ADHD features

\begin{tabular}{lll}
\hline & Average \pm SD & Range \\
\hline Communication & $3.40 \pm 0.54$ & $2.5-4.5$ \\
Social Adaptation & $3.41 \pm 0.54$ & $2.5-4.5$ \\
Empathy & $3.16 \pm 0.67$ & $1.5-5$ \\
Restricted Interests/Behaviors & $3.56 \pm 0.62$ & $2-4.5$ \\
Sensory & $2.39 \pm 0.93$ & $1-4$ \\
Stereotyped/Repetitive Motion & $1.44 \pm 0.55$ & $1-2.5$ \\
Gross Motor & $2.44 \pm 0.85$ & $1-4$ \\
Fine Motor & $2.10 \pm 0.88$ & $1-3.5$ \\
Inattention & $2.84 \pm 0.90$ & $1-5$ \\
Hyperactivity & $1.66 \pm 0.72$ & $1-3.5$ \\
Impulsivity & $2.31 \pm 0.86$ & $1-4$ \\
Sleep Cycle & $2.16 \pm 0.79$ & $1-4$ \\
Learning & $2.09 \pm 0.92$ & $1-4.5$ \\
Language Development & $1.47 \pm 0.71$ & $1-4$ \\
\hline
\end{tabular}

\subsection{Relationship Between Memory and Symptoms}

To clarify the relationship between memory functions and the severity of symptoms, we calculated simple correlations between two index scores from the WMS-R and each domain in the MSPA. As shown in Table 4, Visual Memory correlated with the Fine Motor domain, and Verbal Memory corresponded with the Language Development domain $(r=-0.552, p<0.01 ; r=-0.507, p<0.01$, respectively). Because IQ can contribute to the correlations, we calculated partial correlations controlling for IQ. As shown in Table 5, we found a significant correlation only between Visual Memory and the MSPA score for the Fine Motor domain $(r=-0.581, p<0.01)$ (Figure 1). As shown in Figure 1, nine participants scored within the clinical range for fine motor skills (above 2.5 in the MSPA score) and six obtained borderline scores of 2.5. Thus, as individuals with ASD usually range widely in terms of fine motor skill, nearly half of our participants were also in the borderline or clinically impaired in this regard.

Table 4. Correlations between memory performance and the severity of symptoms

\begin{tabular}{lll}
\hline & Verbal Memory & Visual Memory \\
\hline Communication & -.283 & -.030 \\
Social Adaptation & -0.382 & -.199 \\
Empathy & -0.387 & -.122 \\
Restricted Interests/Behaviors & -0.334 & -.284 \\
Sensory & .005 & -.113 \\
Stereotyped/Repetitive Motion & -.237 & -.222 \\
Gross Motor & -0.336 & -.271 \\
Fine Motor & -.113 & $-.552^{*}$ \\
Inattention & .190 & .061 \\
Hyperactivity & .006 & .111 \\
Impulsivity & .063 & -.082 \\
Sleep Cycle & -.085 & -.066 \\
Learning & -0.406 & -.292 \\
Language Development & $-.507^{*}$ & -.256 \\
\hline
\end{tabular}

Note. ${ }^{*} \mathrm{p}<0.01$ 
Table 5. Partial correlations between memory and the severity of symptoms when IQ was controlled

\begin{tabular}{lll}
\hline & Verbal memory & Visual memory \\
\hline Communication & -.117 & .134 \\
Social Adaptation & -.233 & -.108 \\
Empathy & -.458 & -.171 \\
Restricted Interests/Behaviors & -.215 & -.212 \\
Sensory & -.075 & -.411 \\
Stereotyped/Repetitive Motion & -.240 & -.237 \\
Gross Motor & -.272 & -.275 \\
Fine Motor & -.260 & $-0.581^{*}$ \\
Inattention & -.088 & -.117 \\
Hyperactivity & -.093 & .032 \\
Impulsivity & -.103 & -.133 \\
Sleep Cycle & -.096 & -.101 \\
Learning & -.151 & -.144 \\
Language Development & -.449 & -.133 \\
\hline
\end{tabular}

Note. ${ }^{*} \mathrm{p}<0.01$

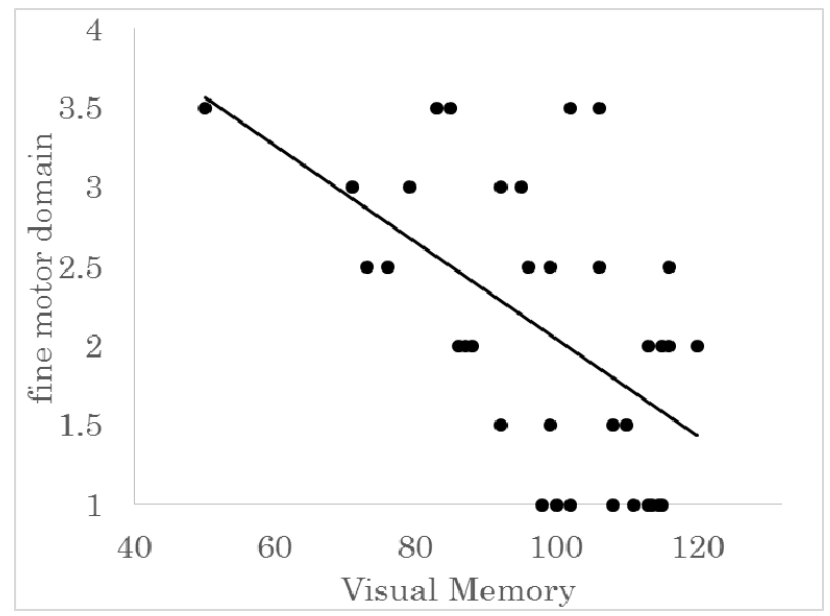

Figure 1. Partial correlation between visual memory in the WMS-R and the fine motor domain in the MSPA, controlling for IQ. We found a significant correlation.

\subsection{Visual Memory and Fine Motor Skills}

Inattention can lead to poor memory performance because of reduced attention to a task, and thus, might also result in decreased fine motor skills, as suggested in the case of people with DAMP (deficits in attention, motor control, and perception) syndrome (Gillberg, 2003). Furthermore, developmental coordination disorder (DCD) is often combined with learning disorder (LD) (Kaplan, Wilson, Dewey, \& Crawford, 1998; Dewey, Kaplan, Crawford, \& Wilson, 2002), which might accompany memory dysfunction. Thus, inattention while learning might be associated with reduced visual memory and fine motor performance. To test this possibility, we conducted a partial correlation analysis that included the MSPA scores for inattention and learning as controlled variables, although they were not correlated with visual memory, as shown in Tables 4 and 5 . We found that the correlation between Visual Memory and the MSPA score for the Fine Motor domain remained significant $(r=$ $-0.661, p<0.001$ ). 
Because the raw scores were not age-adjusted, we calculated the partial correlation between the raw scores from the Visual Memory subtests and the MSPA score for the Fine Motor domain using IQ and age as controlled variables. We found a significant negative correlation between Visual Reproduction and the MSPA score for the Fine Motor domain $(r=-0.528, p<0.01)$, while Visual Paired Associations trended towards a significant correlation with the MSPA score for the Fine Motor domain $(r=-0.417, p<0.05)$. Figure Memory did not significantly correlate with the MSPA score for the Fine Motor domain $(r=-0.219$, ns).

\section{Discussion}

In this study, we investigated the relationship between visual/verbal memory functions and symptoms in individuals with ASD. Our ASD participants had full-IQ scores of almost 100 (99.14 \pm 14.71 ), and average verbal and visual memory scores. Similarly, in most of the previous memory studies of people with ASD, both visual and verbal memory were retained (Minshew, Goldstein, \& Siegel, 1997; Ozonoff \& Strayer, 2001; Boucher, Mayes, \& Bigham, 2012). However, poor visual memory performance has been reported for several tasks, such as facial memory (Williams et al., 2005b), visual working memory (Williams et al., 2005a; Steele et al., 2007), and complex visual memory involving additional processes (Williams et al., 2006; Williams, Minshew, \& Goldstein, 2008). The WMS-R mainly captures basic memory encoding and retrieval, which may explain why our participants performed so well.

We found an association between visual memory and fine motor skills in people with ASD: clumsy individuals showed poor visual memory. ASD is primarily defined by impairments of social cognition and communication. However, fine and gross motor symptoms, which are characteristic features of DCD, are also commonly observed in people with ASD (Dewey, Cantell, \& Crawford, 2007; M. Matson, Matson, \& Beighley, 2011; Sipes, Matson, \& Horovitz, 2011). According to the DSM-IV-TR (American Psychiatric Association, 2000), DCD is characterized by marked impairment of motor coordination that significantly interferes with academic achievement and normal daily activities. For example, children with DCD have bad balance, display clumsiness, and have difficulty writing and drawing (Barnhart, Davenport, Epps, \& Nordquist, 2003).

Our results are compatible with those of previous DCD studies, in which children with DCD have been found to have poor visual and spatial working memory (Tsai, Wilson, \& Wu, 2008; Tsai, Chang, Hung, Tseng, \& Chen, 2012; Chen, Tsai, Hsu, Ma, \& Lai, 2013). Conversely, Crawford and Dewey (2008) reported that not just DCD, but also co-occurring disorders, are associated with visual perceptual problems. Thus, ASD might be the co-occurring disorder in our study. A possible explanation for this association is as follows. During the process of learning motor skills, tracing of visuospatial constellations and limbs trajectories are likely essential. Indeed, a study on visuomotor adaptation in adults without any disorders indicates that people recall the visuomotor map, update this map using spatial working memory resources, and then use this updated map to plan subsequent movements (Anguera, Reuter-Lorenz, Willingham, \& Seidler, 2010). In other words, if visual memory is poor, it might be difficult to execute such motor planning, inhibiting effective control of the limbs. This is compatible with our result that fine motor skills were specifically associated with the Visual Reproduction subtask, where participants needed to retrieve visuospatial information to succeed at the task.

Conversely, impairment of fine motor movements may be primary while poor visual memory is secondary. Fine motor impairments associated with cerebellar dysfunctions are thought to affect cognitive functions such as visuospatial memory (Steinlin, 2008). For instance, children with Dandy-Walker syndrome and hypoplasia of the cerebellum demonstrated poor performance in a task similar to the Visual Reproduction task in our study (Economou \& Katsetos, 2012). In addition, both children with cerebellar lesions and individuals with a cerebellar tumor resection encountered difficulty in the Rey-Osterrieth Complex task (Riva \& Giorgi, 2000; Scott et al., 2001; Steinlin, 2008). Cerebellar pathology is repeatedly suggested to play a role in ASD (Abell et al., 1999; Amaral, Schumann, \& Nordahl, 2008). Thus, cerebellar dysfunction might play a common role in producing poor visual memory in the above-mentioned conditions, including ASD.

Some researchers have proposed that inattention plays a key role in both perceptual and motor deficits in people with ASD, as in the case of DAMP syndrome (Gillberg, 2003). Therefore, as a supplementary analysis, we tested the possible influence of inattention on perceptual and motor parameters. We found no significant effects, as shown in Tables 4 and 5. In our experimental population, the level of inattention did not explain the association between visual memory and motor functions.

Although some members of our ASD population had poor visual memory, as mentioned above, the average performance was sufficient. As shown in Figure 1, we also found that some participants had good visual memory, most of whom were not clumsy. Thus, in the application of our conclusions to a clinical setting, it should be 
noted that inter-individual variability in motor skills and visual memory was substantial in individuals with ASD. To the best of our knowledge, this is the first study to describe the relationship between these factors in people with ASD. Consideration of individual variability might lead to new insights in ASD research. For instance, individuals with ASD who have impaired fine motor skills might benefit from picture indications. Visual assistance is often used to support ASD populations, but it may not be helpful for every individual with ASD. We hope that our results help to elucidate the mechanisms underlying the efficacy of visual support, and thus lead to improvements in the quality of life of people with ASD.

\section{Limitations}

The results of the present study were based on multiple correlations analyses. Although the correlation between fine motor skills and visual memory was independently significant, we believe that further investigations are required.

\section{Conclusion}

Using a comprehensive assessment scale for ASD and ADHD, as well as the well-known memory scale of the WMS-R, we found a relationship between individual variability in fine motor skills and visual memory performance in people with ASD.

\section{Acknowledgements}

This work was supported by a Healthy Labour Science Research Grant for Research on Psychiatric and Neurological Disease and Mental Health (H21-Kokoro-Wakate-021) from the Ministry of Health, Labour, and Welfare, Japan, and the JSPS KAKENHI, Grant Numbers 22791122 and 24119004. We would like to thank all the participants and staff who contributed this study.

\section{References}

Abell, F., Krams, M., Ashburner, J., Passingham, R., Friston, K., Frackowiak, R., \& Frith, U. (1999). The neuroanatomy of autism: A voxel-based whole brain analysis of structural scans. NeuroReport, 10, 1647-1651. http://dx.doi.org/10.1097/00001756-199906030-00005

Amaral, D. G., Schumann, C. M., \& Nordahl, C. W. (2008). Neuroanatomy of autism. Trends in Neurosciences, 31, 137-145. http://dx.doi.org/10.1016/j.tins.2007.12.005

Ambery, F. Z., Russell, A. J., Perry, K., Morris, R., \& Murphy, D. G. M. (2006). Neuropsychological functioning in adults with Asperger syndrome. Autism, 10, 551-564. http://dx.doi.org/10.1177/ 1362361306068507

American Psychiatric Association. (2000). Diagnostic and statistical Manual of mental disorders-fourth edition-text revision (DSM-IV-TR). Washington, DC: American Psychiatric Press. http://dx.doi.org $/ 10.1111 /$ jsm. 12379

Anguera, J. A., Reuter-Lorenz, P. A., Willingham, D. T., \& Seidler, R. D. (2010). Contributions of spatial working memory to visuomotor learning. Journal of Cognitive Neuroscience, 22, 1871-1930. http://dx.doi.org/10.1162/jocn.2009.21351

Barnhart, R. C., Davenport, M. J., Epps, S. B., \& Nordquist, V. M. (2003). Developmental Coordination Disorder. Physical Therapy, 83, 722-731.

Bemporad, J. R. (1979). Adult recollections of a formerly autistic child. Journal of Autism and Developmental Disorders, 9, 179-197. http://dx.doi.org/10.1007/BF01531533

Bennetto, L., Pennington, B. F., \& Rogers, S. J. (1996). Intact and impaired memory function in Autism. Child Development, 67, 1816-1835. http://dx.doi.org/10.2307/1131734

Boucher, J., Mayes, A., \& Bigham, S. (2012). Memory in Autistic Spectrum Disorder. Psychological Bulletin, 138, 458-496. http://dx.doi.org/10.1037/a0026869

Bowler, D. M., Gardiner, J. M., \& Grice, S. J. (2000). Episodic memory and remembering in adults with Asperger Syndrome. Journal of Autism and Developmental Disorders, 30, 295-304. http://dx.doi.org/ 10.1023/A:1005575216176

Chen, I., Tsai, P., Hsu, Y., Ma, H., \& Lai, H. (2013). Everyday memory in children with developmental coordination disorder. Research in Developmental Disabilities, 34, 687-694. http://dx.doi.org/10.1016/ j.ridd.2012.09.012 
Cohen, S. (1998). Targeting autism: What we know, don't know, and can do to help young children with autism. Berkley, CA: University of California Press.

Crawford, S. G., \& Dewey, D. (2008). Co-occurring disorders: A possible key to visual perceptual deficits in children with developmental coordination disorder? Human Movement Science, 27, 154-169. http://dx.doi.org/10.1016/j.humov.2007.09.002.

Dawson, G., Meltzoff, A. N., Osterling, J., \& Rinaldi, J. (1998). Neuropsychological correlates of early symptoms of autism. Child Development, 69, 1276-1285. http://dx.doi.org/10.2307/1132265

Dewey, D., Cantell, M., \& Crawford, S. G. (2007). Motor and gestural performance in children with autism spectrum disorders, developmental coordination disorder, and/or attention deficit hyperactivity disorder. Journal of the International Neuropsychological Society, 13, 246-256. http://dx.doi.org/10.1017/ S1355617707070270

Dewey, D., Kaplan, B. J., Crawford, S. G., \& Wilson, B. N. (2002). Developmental Coordination Disorder: Associated problems in attention, learning, and psychosocial adjustment. Human Movement Science, 21, 905-918. http://dx.doi.org/10.1016/S0167-9457(02)00163-X

Economou, A., \& Katsetos, C. D. (2012). Patterns of cognitive and fine motor deficits in a case of Dandy-Walker continuum. Journal of Child Neurology, 27, 930-937. http://dx.doi.org/10.1177 /0883073811429500

Funabiki, Y., Kawagishi, H., Uwatoko, T., Yoshimura, S., \& Murai, T. (2011). Development of a multi-dimensional scale for PDD and ADHD. Research in Developmental Disabilities, 32, 995-1003. http://dx.doi.org/10.1016/j.ridd.2011.01.052

Gillberg, C. (2003). Deficits in attention, motor control, and perception: A brief review. Archives of Disease in Childhood, 88, 904-910. http://dx.doi.org/10.1136/adc.88.10.904

Kanner, L. (1943). Autistic disturbances of affective contact. Nervous Child, 2, 217-250.

Kaplan, B. J., Wilson, B. N., Dewey, D., \& Crawford, S. G. (1998). DCD may not be a discrete disorder. Human Movement Science, 17, 417-490. http://dx.doi.org/10.1016/S0167-9457(98)00010-4

Koshino, H., Carpenter, P. A., Minshew, N. J., Cherkassky, V. L., Keller, T. A., \& Just, M. A. (2005). Functional connectivity in an fMRI working memory task in high-functioning autism. Neuroimage, 24, 810-821. http://dx.doi.org/10.1016/j.neuroimage.2004.09.028

Matson, J. L., \& Nebel-Schwalm, M. S. (2007). Comorbid psychopathology with Autism Spectrum Disorder in children: An overview. Research in Developmental Disabilities, 28, 341-352. http://dx.doi.org/10.1016/ j.ridd.2005.12.004

Matson, M. L., Matson, J. L., \& Beighley, J. S. (2011). Comorbidity of physical and motor problems in children with autism. Research in Developmental Disabilities, 32, 2304-2308. http://dx.doi.org/10.1016/ j.ridd.2011.07.036

Minshew, N. J., Goldstein, G., \& Siegel, D. J. (1997). Neuropsychologic functioning in autism: Profile of a complex information processing disorder. Journal of the International Neuropsychological Society, 3, 303-316.

Ozonoff, S., \& Strayer, D. L. (2001). Further evidence of intact working memory in autism. Journal of Autism and Developmental Disorders, 31, 257-263. http://dx.doi.org/10.1023/A:1010794902139

Renner, P., Klinger, L. G., \& Klinger, M. R. (2000). Implicit and explicit memory in autism: Is autism an Amnesic Disorder? Journal of Autism and Developmental Disorders, 30, 3-14. http://dx.doi.org/10.1023/ A:1005487009889

Riva, D., \& Giorgi, C. (2000). The cerebellum contributes to higher functions during development: Evidence from a series of children surgically treated for posterior fossa tumours. Brain, 123, 1051-1061. http://dx.doi.org/10.1093/brain/123.5.1051

Scott, R. B., Stoodley, C. J., Anslow, P., Paul, C., Stein, J. F., \& Sugden, E. M. (2001). Lateralized cognitive deficits in children following cerebellar lesions. Developmental Medicine \& Child Neurology, 43, 685-691. http://dx.doi.org/10.1017/S0012162201001232 
Siegel, D. J., Minshew, N. J., \& Goldstein, G. (1996). Wechsler IQ profiles in diagnosis of high-functioning autism. Journal of Autism and Developmental Disorders, 26, 389-406. http://dx.doi.org/10.1007/ BF02172825

Sipes, M., Matson, J. L., \& Horovitz, M. (2011). Autism spectrum disorders and motor skills: The effect on socialization as measured by the Baby And Infant Screen For Children with a Utism Traits (BISCUIT). Developmental Neurorehabilitation, 14, 290-296. http://dx.doi.org/10.3109/17518423.2011.587838

Spek, A. A., Scholte, E. M., \& van Berckelaer-Onnes, I. A. (2008). Brief report: The Use of WAIS-III in adults with HFA and Asperger Syndrome. Journal of Autism and Developmental Disorders, 38, 782-787. http://dx.doi.org/10.1007/s10803-007-0446-5

Steel, G., Gorman, R., \& Flexman, J. E. (1984). Neuropsychiatric testing in an autistic mathematical idiot-savant: Evidence for nonverbal abstract capacity. Journal of the American Academy of Child Psychiatry, 23, 704-707. http://dx.doi.org/10.1016/S0002-7138(09)60540-9

Steele, S. D., Minshew, N. J., Luna, B., \& Sweeney, J. A. (2007). Spatial working memory deficits in autism. Journal of Autism and Developmental Disorders, 37, 605-612. http://dx.doi.org/10.1007/ s10803-006-0202-2

Steinlin, M. (2008). Cerebellar disorders in childhood: Cognitive problems. Cerebellum, 7, 607-610. http://dx.doi.org/10.1007/s12311-008-0083-3

Sugishita, M. (2001). Japanese Wechsler Memory Scale-Revised. Tokyo: Nihonbunkakagakusha.

Tsai, C., Chang, Y., Hung, T., Tseng, Y., \& Chen, T. (2012). The neurophysiological performance of visuospatial working memory in children with developmental coordination disorder. Developmental Medicine \& Child Neurology, 54, 1114-1120. http://dx.doi.org/10.1111/j.1469-8749.2012.04408.x

Tsai, C., Wilson, P. H., \& Wu, S. K. (2008). Role of visual-perceptual skills (non-motor) in children with developmental coordination disorder. Human Movement Science, 27, 649-664. http://dx.doi.org/10.1016/ j.humov.2007.10.002

Wechsler, D. (1987). Wechsler memory scale manual revised. San Antonio: The Psychological Corporation.

Wechsler, D. (1991). Manual for the Wechsler intelligence scale for children (3rd ed.). San Antonio, TX: The Psychological Corporation.

Wechsler, D. (1997). The Wechsler adult intelligence scale (3rd ed). San Antonio, TX: The Psychological Corporation.

Williams, D. L., Goldstein, G., \& Minshew, N. J. (2005b). Impaired for faces and social scenes in autism: Clinical implications of memory dysfunction. Archives of Clinical Neuropsychology, 20, 1-15. http://dx.doi.org/10.1016/j.acn.2002.08.001

Williams, D. L., Goldstein, G., \& Minshew, N. J. (2006). The profile of memory function in children with autism. Neuropsychology, 20, 21-29. http://dx.doi.org/10.1037/0894-4105.20.1.21

Williams, D. L., Goldstein, G., Carpenter, P. A., \& Minshew, N. J. (2005a). Verbal and spatial working memory in autism. Journal of Autism and Developmental Disorders, 35, 747-756. http://dx.doi.org/10.1007/ s10803-005-0021-x

Williams, D. L., Minshew, N. J., \& Goldstein, G. (2008). Memory within a complex information processing model of autism. In J. Boucher, \& D. Bowler (Eds.), Memory in Autism: Theory and Evidence (pp. 125-142). Cambridge, England: Cambridge University Press. http://dx.doi.org/10.1017/ CBO9780511490101.009

\section{Copyrights}

Copyright for this article is retained by the author(s), with first publication rights granted to the journal.

This is an open-access article distributed under the terms and conditions of the Creative Commons Attribution license (http://creativecommons.org/licenses/by/3.0/) 